\title{
RESULTS FROM THE SCALED FINAL FOCUS EXPERIMENT
}

\author{
S. A. MacLaren, M. de Hoon, A. Faltens, W. Ghiorso, P. Seidl, Lawrence Berkeley \\ National Laboratory, Berkeley, CA 94720
}

\begin{abstract}
:
Vacuum ballistic focusing is the straightforward method to obtain a heavy ion beam spot size necessary to drive an inertial confinement fusion target. The beam is first expanded then focused to obtain the desired convergence angles at the exit of the last element. This is done in an attempt to achieve a focal spot size in which emittance is the limiting factor; however, aberrations and space charge will influence the spot radius. Proper scaling of particle energy, mass, beam current, beam emittance, and magnetic field replicates the dynamics of a full driver beam at the focus in a small laboratory experiment. By scaling the beam current to $\sim 100 \mu \mathrm{A}, 160 \mathrm{keV} \mathrm{Cs}^{+}$has been used to study experimentally a proposed driver design at one-tenth scale. Once a nominal focal spot is achieved, the magnet strengths are deliberately de-tuned to simulate the effect of an off-momentum slice of the beam. Additionally, several methods will be used to inject electrons into beam following the last focusing element in order to study the neutralization of space charge and its effect on the focus. Transverse phase space and beam current density measurements at various stages of the focus will be presented as well spot size measurements from the various trials. This data will be compared to the results of a PIC model of the experiment.
\end{abstract}

Corresponding Author: Stephan MacLaren, MS 47-112, Lawrence Berkeley

National Laboratory, One Cyclotron Road, Berkeley, CA 94720

Phone: 5104864245

Fax: 5104865392

Email: samaclaren@lbl.gov 


\section{BACKGROUND AND MOTIVATION}

The design of the final focus for a heavy ion fusion power plant must be the result of careful merger of driver and chamber issues of physics, engineering, and cost. The array of available options must be compared critically in order to select an approach that will be both feasible and cost effective. The choices for a final focus/chamber transport subsystem can be placed into three categories based on the types of physics involved: unneutralized (high vacuum) ballistic focusing, ballistic focusing with neutralization, and other forms of non-ballistic focusing generally referred to as channel transport. The three are listed in what is generally accepted to be in order of increasing uncertainty as far as

physics issues are concerned. On the other hand, they are in decreasing order in terms of the constraints placed on the output of the accelerator, which directly effect the cost of electricity.

The Scaled Final Focus Experiment at Berkeley Laboratory is based on the HIBALL-II ${ }^{1}$ final focus subsystem design; both the design and experiment consist of a set of six magnetic quadrupoles to ballistically focus an ion beam to a small spot. By properly scaling the physics parameters that relate particle energy and mass, beam current, beam emittance, and focusing field, we replicate the dynamics of a full driver beam in a small laboratory beam. This subsystem design falls into the first of the above categories and was part of the overall HIBALL-II report that included a $4 \mathrm{GW}$ electric output, benefiting from economy of scale. More recent cost-effective designs, however, prefer lower energy ion beams and close coupled, higher yield targets in smaller plants. 
By increasing the perveance of the experimental beam and introducing a source of electrons, we study a neutralized focus, with scaling applicable to these types of drivers.

\section{SCALING OF THE EXPERIMENT}

As mentioned above, in order that the measurements made on the experiment be meaningful, it is necessary to accurately reproduce the dynamics of the driver design. Specifically, this is done by identifying the parameters that are limited by the available experimental facilities, and adjusting the remaining parameters to preserve the physics of interest. For our experiment, the physics of interest for scaling purposes are contained in the relation for the horizontal envelope of the beam

$$
\frac{d^{2} a}{d z^{2}}=-k_{x} a+\frac{2 Q}{(a+b)}+\frac{\varepsilon_{x}^{2}}{a^{3}}
$$

where $a$ and $b$ are the beam semi-axes, $k_{x}$ is the external horizontal focusing gradient, $Q$ is the dimensionless perveance, and $\varepsilon_{x}$ is the horizontal emittance. A similar relation can be written for the vertical. The parameters fixed by the facilities are the physical dimensions of the experiment, for which we have chosen a one-tenth scale, the kinetic energy of the ions, which is $160 \mathrm{KeV}$ as compared to the $10 \mathrm{GeV}$ of the design, and the emittance, which is primarily determined by the ion source. These three are not entirely independent; the emittance is affected by the dimensional scaling, and it was therefore important to select a source design that produced a very low emittance for use in this much smaller scale experiment. Finally, the parameters that are adjusted in order to reproduce the beam dynamics are the beam current, and the strengths of the focusing fields. 
To use the envelope equation as a guide for proper scaling, we first note that the equation has dimensions of inverse length. Therefore, each of the terms in the equation needs to be adjusted by the inverse of the dimensional scale factor:

$\frac{\text { term }_{e}}{\text { term }_{d}}=\frac{\text { length }_{d}}{\text { length }_{e}}=D=10$

where the subscript $e$ refers to the experiment and $d$ to the design. First we scale gradient term to derive the relation for the magnetic field

$$
\frac{k_{e} a_{e}}{k_{d} a_{d}}=\frac{\left(B_{e} / a_{e}\right)(m \beta \gamma)_{d}}{\left(B_{d} / a_{d}\right)(m \beta \gamma)_{e}} \frac{a_{e}}{a_{d}}=D
$$

Repeating the process for the other terms,

$$
\frac{Q_{e} / a_{e}}{Q_{d} / a_{d}}=\frac{I_{e}\left(m \beta^{3} \gamma^{3}\right)_{d}}{I_{d}\left(m \beta^{3} \gamma^{3}\right)_{e}} \frac{a_{d}}{a_{e}}=D
$$

$\frac{\varepsilon_{e}^{2} / a_{e}^{3}}{\varepsilon_{d}^{2} / a_{d}^{3}}=D$

yields scaling relations for the beam current and emittance. The experiment-to-design ratios for these parameters can then be solved for explicitly, keeping in mind that the ratio of the design radius to the experimental radius is just $\mathrm{D}$, the dimensional scaling factor.

The effect of the scaling is to produce a set of manageable parameters for a small scale experiment. The HIBALL-II design used $\mathrm{Bi}^{+}$ions (mass 209) at $10 \mathrm{GeV}$ and has a $\beta$ of 0.32 . The experiment uses $\mathrm{Cs}^{+}$at $160 \mathrm{keV}$; therefore, the experimental $\beta$ is 200 times smaller than that of the design. To preserve the perveance, the current is scaled from $1.25 \mathrm{kA}$ to $95 \mu \mathrm{A}$, and the magnet fields need only be a few hundred gauss. Because the beam trajectories are similar, $9 \mathrm{mr}$ is the final convergence angle for both cases. These results, as well as the explicit scaling relations for the parameters are summarized in Table 1. 


\section{DESCRIPTION OF THE EXPERIMENT}

The ion source presently in use was specifically designed with the scaled experiment in mind. The emitter is a contact ionizer made by sintering iridium powder, and is periodically doped with a solution of $\mathrm{Cs}_{2} \mathrm{CO}_{3}$, which decomposes at low temperatures to leave a fractional monolayer of $\mathrm{Cs}^{+}$on the surfaces of the iridium particles. It operates at $1400 \mathrm{~K}$ and is surrounded by an array of heat shields for maximum thermal efficiency. The package is inserted into a Pierce electrode and a current density of $8 \mathrm{~mA} / \mathrm{cm}^{2}$ is extracted through an opening in a flat anode plate. The source design and performance are described by MacLaren et. al. ${ }^{2}$

An aperture immediately after the diode selects the beam current to be used in the experiment. Following the aperture is a 1.5 meter lattice of ten electrostatic quadrupoles. This lattice serves to match the apertured beam size and convergence angle into the drift preceding the final focus lattice. The two sets of slit scanner diagnostics that serve this section are after the fifth and tenth electrostatic elements.

The magnetic focusing section is 8.2 meters long, including initial and final drifts, and includes slit scanner diagnostics after the third and sixth magnets. The middle drift between the third and fourth magnets has been shortened with respect to the HIBALL-II design as the bending magnets that allow for the neutron dump are not included. The quadrupole magnets consist of two layers of copper windings in a $\cos (2 \theta)$ distribution on cylindrical shells, powered by pulsers synchronized with the beam pulse. Each of the magnet inner diameters is lined with a grounded metal foil to simulate the beam pipe in the design focusing system. 
A schematic of the experiment along with the beam envelope is shown in Figure 1. The upper part of the figure contains a to-scale drawing of the vacuum system with electrostatic elements and magnetic elements shaded. The lower part is a representation of the horizontal and vertical beam envelopes with the transverse scale exaggerated. The dashed lines represent the locations of the diagnostic probes. The envelope displayed in the figure represents the lower perveance $(95 \mu \mathrm{A})$ case in which only the last four electrostatic quadrupoles are used. In a higher current case in which a neutralized focus is examined, all ten of these quadrupoles are employed to carefully match the higher perveance beam into the magnetic focusing lattice.

To set the magnet currents, a code based on the envelope equation determines the field gradients necessary to give the beam the desired envelope parameters. (The same code is used to solve for the quadrupole voltages in the electrostatic section.) These gradients are then used in a simple calculation of the necessary peak pulser currents that assumes a continuous sheet of current with a $\cos (2 \theta)$ azimuthal distribution at a radius between the two concentric shells of windings. The beam parameters are then measured experimentally and fed back to the code; because the code contains a graphical user interface, it is relatively easy to adjust the focusing gradients to fit the measured parameters. The adjustments are applied to the magnet pulsers and the measurements are repeated. There is no straightforward means in place to directly measure the pole tip fields of the magnets, but this iterative procedure provides an efficient and effective way to match the desired envelope trajectories.

Each diagnostic location consists of at least one pair of automated slit scanner probes. A single slit is used to measure the transverse current density profile of the beam. 
Parallel slits are used to measure the phase space density and emittance of the beam. Slits oriented perpendicular to each other can be used to map out the current density in the X-Y plane (the "crossed slit" measurement). Each of these measurements is completely automated, using LabView ${ }^{\mathrm{TM}}$ software to trigger the source diode pulse, move the probes, and extract and store the measured waveforms from a digitizing oscilloscope.

In addition to the slit scanners, there are two Faraday cups for measurement of the total beam current and transmission through the final focus lattice; one that can be inserted in place of the $7^{\text {th }}$ and $8^{\text {th }}$ electrostatic quadrupoles, and another after the last magnet. Co-located with this second Faraday cup is a capacitive drift tube diagnostic that is used to measure the total charge of the incident beam. Finally, $80 \mathrm{~cm}$ downstream of the last magnet is the focal spot probe. This probe consists of two shallow Faraday cups, each masked with .002" slits oriented to perform current density scans in both transverse directions. The cup assembly is mounted on a platform that translates along all three axes, such that beam spot profiles can be measured at various axial locations within a 15 $\mathrm{cm}$ range. Biased guard rings are mounted in front of the slits in order to prevent secondary electrons from streaming back up the beam path and partially neutralizing the remaining beam.

\section{4. $95 \mu \mathrm{A}$ FOCUS}

A crossed slit measurement of the beam is made at the first diagnostic station; at this point the beam has simply drifted and expanded from the aperture. The current density is shown in Figure 2, and inspection of the data shows that the "flat top" of the beam begins to fall off at $\sim 4.5 \mathrm{~mm}$ and has a width of $\sim 2 \mathrm{~mm}$. A Debye length of 0.3 
mm can be calculated using the line charge density at this location, and using the transverse emittance to determine the temperature of the beam. With a ratio of $\lambda_{D} / a$ typical of a space charge dominated beam, one expects a fairly flat profile with the top extending to $\sim 70 \%$ of the radius, in agreement with the data.

Simulations of the experiment in various configurations have been performed using a 2D particle in cell code (WARP). Measurements of the phase space at the various diagnostic locations can be compared with the WARP results using contour plots to represent the phase space density. A detailed report on the results of these simulations can be found in these proceedings. ${ }^{3}$ The vertical transverse phase space of the converging beam at the exit of the last magnet is shown in Figure 3. It is often insightful to shear the phase space data, zeroing the cross moment, in order to study the distortions of the phase space ellipse. Plotted also are sheared versions of the measured phase space and the WARP results at this location.

Multiplying the envelope equation by $d a / d z$ and integrating from the end of the last magnet to the focus produces a relation for the focal spot size

$$
2 Q \cdot \ln \frac{a_{o}}{a_{f}}=\theta^{2}-\left(\frac{\varepsilon^{2}}{a_{f}^{2}}-\frac{\varepsilon^{2}}{a_{o}^{2}}\right)
$$

where $a_{o}$ and $\theta$ represent the envelope radius and convergence angle at the exit of the last magnet and $a_{f}$ is the spot size. Solving for the spot size graphically, we find an expected radius of $0.65 \mathrm{~mm}$ for the parameters measured at $\mathrm{D} 4$. It is also important to note that at the focal point, the ratio of the emittance term to the perveance term in the envelope equation is 5.8 for this calculated radius. We should therefore expect to measure a Gaussian profile characteristic of an emittance dominated focus. 
Figure 4 shows the results of horizontal and vertical scans taken with the 3 -axis probe at the same axial location. The results of the Gaussian fit show that the beam is almost round with a twice rms radius of $0.5 \mathrm{~mm}$. This is smaller that the $0.65 \mathrm{~mm}$ value calculated using the parameters measured at the D4 location. Alternately, using the twotimes-rms radius from the second moment of the raw data, the horizontal radius is 0.75 $\mathrm{mm}$ and the vertical radius is $0.57 \mathrm{~mm}$. These results bracket the calculated value. The figure also plots the normalized signal integrated as a function of distance from the centroid of the distribution. This yields the fraction of the ions that strike within a given distance from the center, projected onto either the horizontal or vertical axis. On these plots the results of the WARP simulation at the focal spot are also presented. To achieve a valid comparison with the measurement, the macro-particles are collapsed onto a transverse axis and binned at $100 \mu \mathrm{m}$ intervals. The bins are integrated in the same manner as the data and plotted as the green curve. To compare these results with the HIBALL-II design, a Gaussian profile is assumed for the focal spot, and the twice rms spot radius calculated from Equation $6(0.58 \mathrm{~mm})$ is assigned as $2 \sigma$. The profile is projected onto the transverse axis, and the integrated current versus position is represented by the darkest curve.

It may be noted that the shape of the beam envelope measured in the first half of the magnetic section is different from the envelope depicted in the design report. The HIBALL-II accelerator delivered a beam to the final focus section with a radius considerably smaller that the average radius through the first three magnets. The beam was allowed to drift expand in the section preceding the first magnet. Initially, the scaled experiment adopted this approach by keeping the beam at a $2-3 \mathrm{~mm}$ radius through the 
electrostatic section, then allowing it to expand in the drift to an average radius of $17 \mathrm{~mm}$ in the magnet section. This solution resulted in a ten-fold growth in emittance between measurements at D2 and D4. This emittance growth was not a result of excessive distortions in the phase space, but more as a fairly uniform expansion of the phase space area, as shown in Figure 5. This emittance growth did not appear in the WARP simulation, and its cause is still under investigation.

\section{5. $400 \mu \mathrm{A}$ FOCUS}

To study the effect of neutralization on the focus, an increase in the beam perveance was necessary in order to push the focus into the space charge-dominated regime. This allows for an easily measurable effect on the spot size provided a significant fraction of the beam can be neutralized. A larger diameter aperture was used at the source to select $400 \mu \mathrm{A}$; this is the focusable current limit for the experiment keeping the final convergence angle fixed without altering the diameters and strengths of the focusing elements or the drift lengths. With this beam, the ratio of the emittance to space charge terms for an un-neutralized focus is 0.2 , as compared to 7 if the beam is neutralized to $80 \%$. The change in the measured focal spot should be clearly recognizable as the focus moves from the space charge-dominated to the emittancedominated regime.

Two different methods were employed to try to introduce co-moving electrons

into the beam. A cold, biased, stainless steel grid was inserted into the beam path at the location of the D4 diagnostic probes to produce secondary electrons from glancing collisions with the beam ions. The secondary electrons are born with the several eV energies, and this method had been used successfully ( $<90 \%$ neutralization) in a previous 
experiment with a much higher current beam. Because an electron only needs an energy of $0.6 \mathrm{eV}$ in order to match the $\beta$ of the beam, only a small fraction of the secondaries will have the axially oriented momentum component to allow them to be co-moving with the ions. Additionally, the potential of the final focus beam from edge to center is only $7.5 \mathrm{~V}$, providing a very shallow well in which to trap the electrons' transverse motion. A measurement of the beam current downstream of the grid using the drift tube probe indicated that the beam had been neutralized by a maximum of $40 \%$ when a bias of $-1 \mathrm{~V}$ was applied. By biasing the grid to $\sim+25 \mathrm{~V}$, the neutralizing effect could be completely removed as the secondary electrons were forced back to the grid. Measurements of the resulting spot radius were in agreement with a $40 \%$ neutralization factor.

The second method involved placing a hot $(2100 \mathrm{~K})$ filament of $76 \mu \mathrm{m}$ tungsten wire into the path of the beam, again at the D4 location. The wire is powered by a DC circuit that turns off the filament current $500 \mu \mathrm{sec}$ before the source is pulsed. This gives the voltage across the filament enough time to fall to zero by the time the beam arrives, with the filament remaining hot enough to provide copious amounts of electrons with energies of a few tenths of an $\mathrm{eV}$. Again, the drift tube diagnostic was used first, and measured a neutralization fraction of $65 \%$. The beam spot measurements, when compared to envelope calculations at reduced perveances, indicate that the fraction is closer to $80 \%$. It is suspected that as the line charge density of the beam is significantly reduced, the drift tube signal, which must be integrated in order to make a comparison with the beam current trace, is a less accurate measurement. In particular, at higher fractional neutralization, the bulk of the line charge appears in the fast current spike at the 
head of the beam, and the integrated signal is affected more strongly by the time resolution of the amplifier.

The full-perveance and neutralized spot measurements are displayed in Figure 6. The radius of the un-neutralized beam spot is $3.1 \mathrm{~mm}$, and the injection of electrons shrinks the radius to $1.1 \mathrm{~mm}$ and produces the Gaussian profile characteristic of an emittance dominated focus.

Finally, a study of the effect of an off-momentum beam on the focus was performed using the neutralized focal spot. Because the focusing force in the magnets depends linearly on the momentum of the ions, a momentum shift may be simulated in the experiment by deliberately de-tuning the magnet strengths. This method was used to measure the results of $\pm 0.5 \%$ and $\pm 1.0 \%$ deviations in the momentum of the beam. The smaller momentum shift produced a spots that were $\sim 20 \%$ larger, while the larger shifts caused increases of $\sim 50 \%$.

\section{CONCLUSIONS AND FUTURE EFFORT}

The Scaled Final Focus Experiment has accomplished its original goal of testing many of the important beam physics issues associated with a power plant system that employs a ballistic focus. From the results of the experiment, it appears that this type of scheme is validated, provided the accelerator and reactor chamber parameters are achievable. In addition, we have been able to use the same experimental apparatus to test some of the issues fundamental to more recent power plant schemes that employ higher perveance beams. A neutralized focus is necessary for these designs to work without resorting to an unwieldy number of beams. These results indicate that injection of comoving electrons is a viable approach to neutralization and further study is merited. 
To complete this work, it is desirable to continue comparisons of the data to the 2D WARP simulations, as well as to construct a 3D WARP model of the experiment. In particular, the nature of the phase space distortions measured in the experiment may be further understood with a more accurate modeling of the magnetic fields. The unexpected and considerable emittance growth observed in the attempt to exactly match the shape HIBALL-II beam envelope will also be studied further, both experimentally and in simulation.

\section{ACKNOWLEDGEMENTS}

The authors gratefully acknowledge the outstanding support of the LBNL technical staff, including Mr. R. Beggs, Mr. R. Hipple, Mr. C. Rogers, and Mr. W. Strelo. This work is supported by the Director, Office of Energy Research, Office of Fusion Energy Science, US DOE, under Contract \# DE-AC03-76SF00098. 


\section{PLEASE NOTE: FIGURES 1 AND 4 ARE 2-COLUMN OBJECTS. ALL OTHER \\ FIGURES AND THE TABLE ARE 1-COLUMN OBJECTS.}

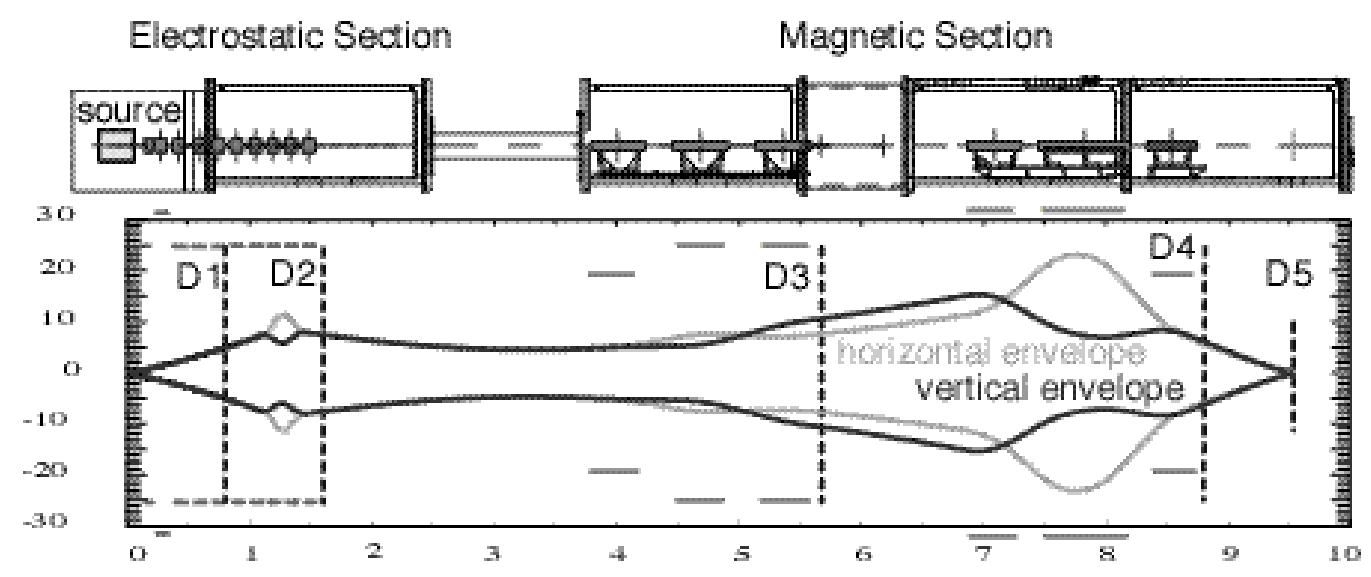

Figure 1

Figure 1: A to-scale schematic of the experiment is shown above a plot of the horizontal and vertical envelopes for the $95 \mu \mathrm{A}$ beam. Diagnostic locations are indicated by vertical dashed lines, and the focusing element apertures are indicated by horizontal bars.

D1 current density

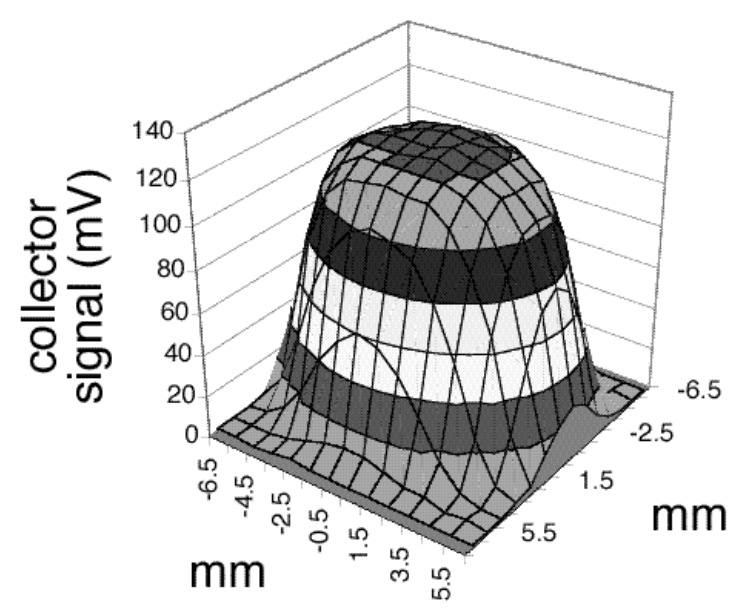

Figure 2: A crossed-slit current density map of the $95 \mu \mathrm{A}$ beam at the first diagnostic station. Shown is a time slice characteristic of the steady state region in the 
middle of the pulse. The profile is characteristic of a space charge dominated beam $\left(\lambda_{\mathrm{D}} / \mathrm{a}\right.$ $=0.05)$

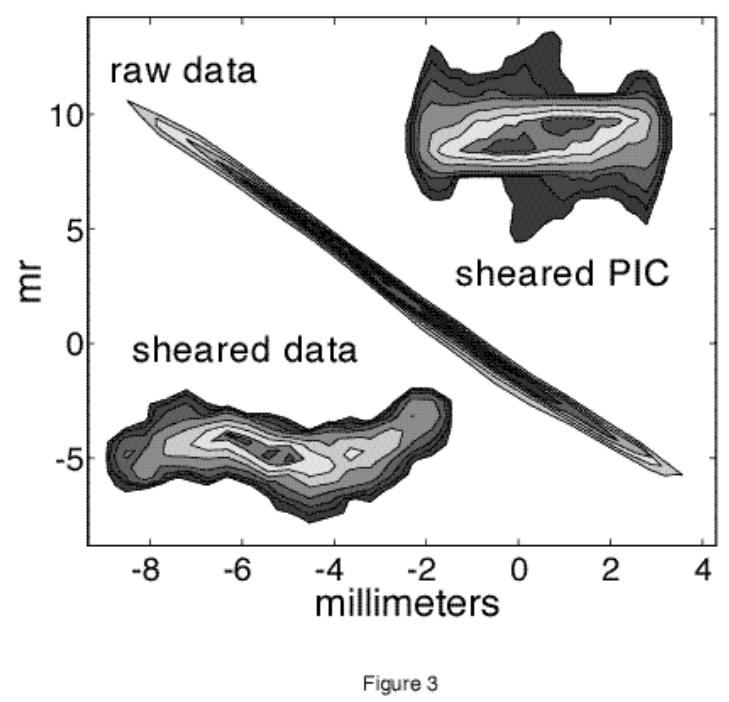

Figure 3: The central plot is the $\mathrm{Y}-\mathrm{Y}^{\prime}$ phase space of the $95 \mu \mathrm{A}$ beam at the exit of the last magnet. Above and below are sheared versions of the same phase space for the real beam and the WARP simulation, respectively. 

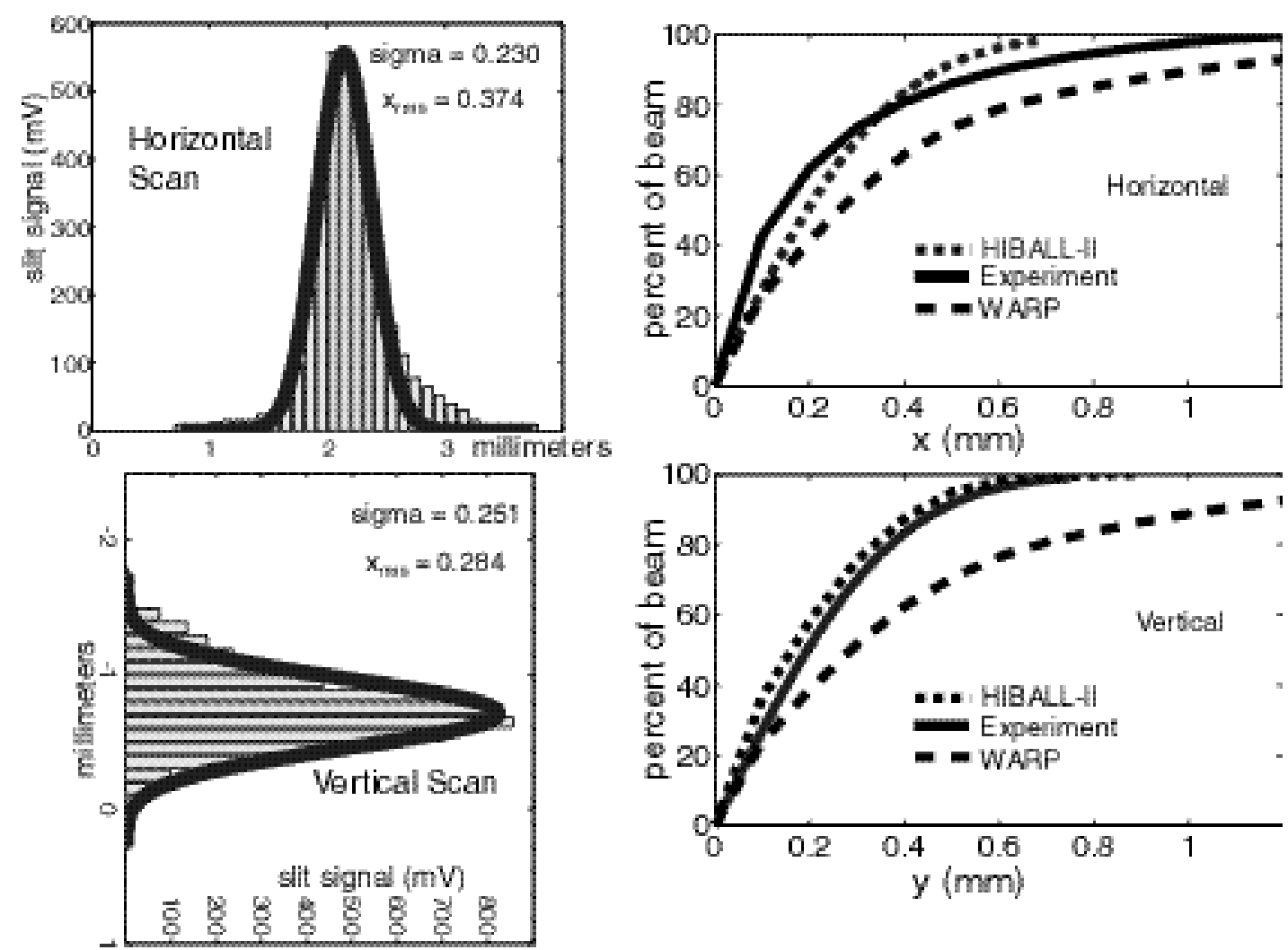

Figure 4: The figures on the left show Gaussian curves fitted to the spot profile

data. Each is a time slice characteristic of the steady state region in the middle of the

pulse. The other figures plot percent of measured beam current as a function of distance from the centroid. The solid curve plots the scaled HIBALL-II result $(2 \sigma=0.58 \mathrm{~mm})$ projected onto the transverse axis. 


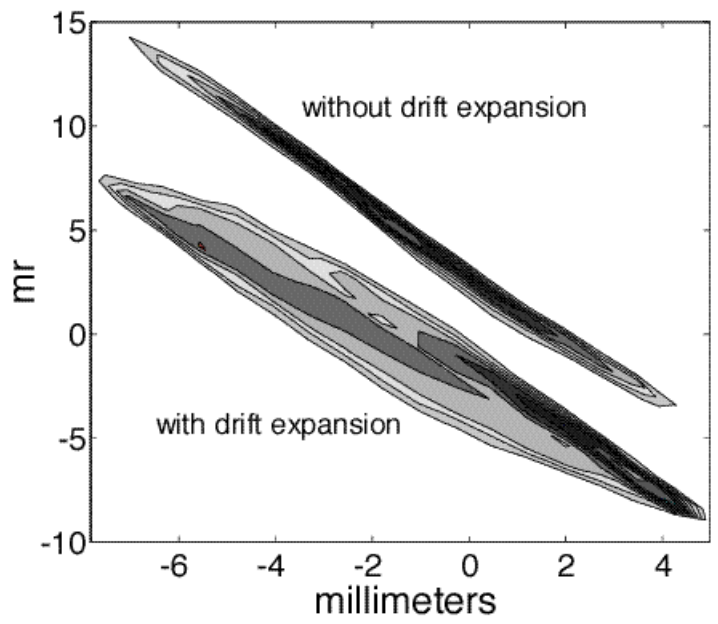

Figure 5

Figure 5: Two plots of $\mathrm{Y}^{-\mathrm{Y}^{\prime}}$ phase space data at D4 are shown at the same scale. The lower set of contours demonstrates the inflation of the phase space area for the beam that undergoes an initial drift expansion. 

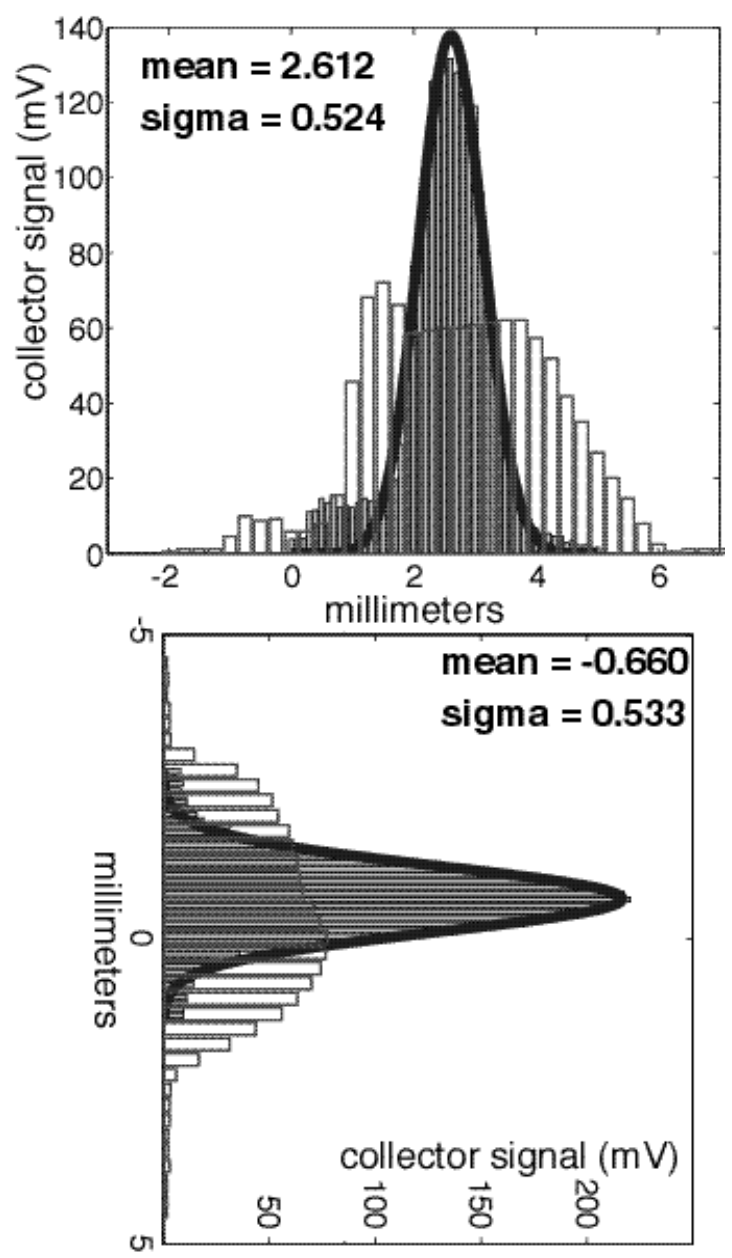

Figure 6

Figure 6: Gaussian curves are fitted to time slices of the neutralized spot profile data. The un-neutralized data is shown with hollow bars. 


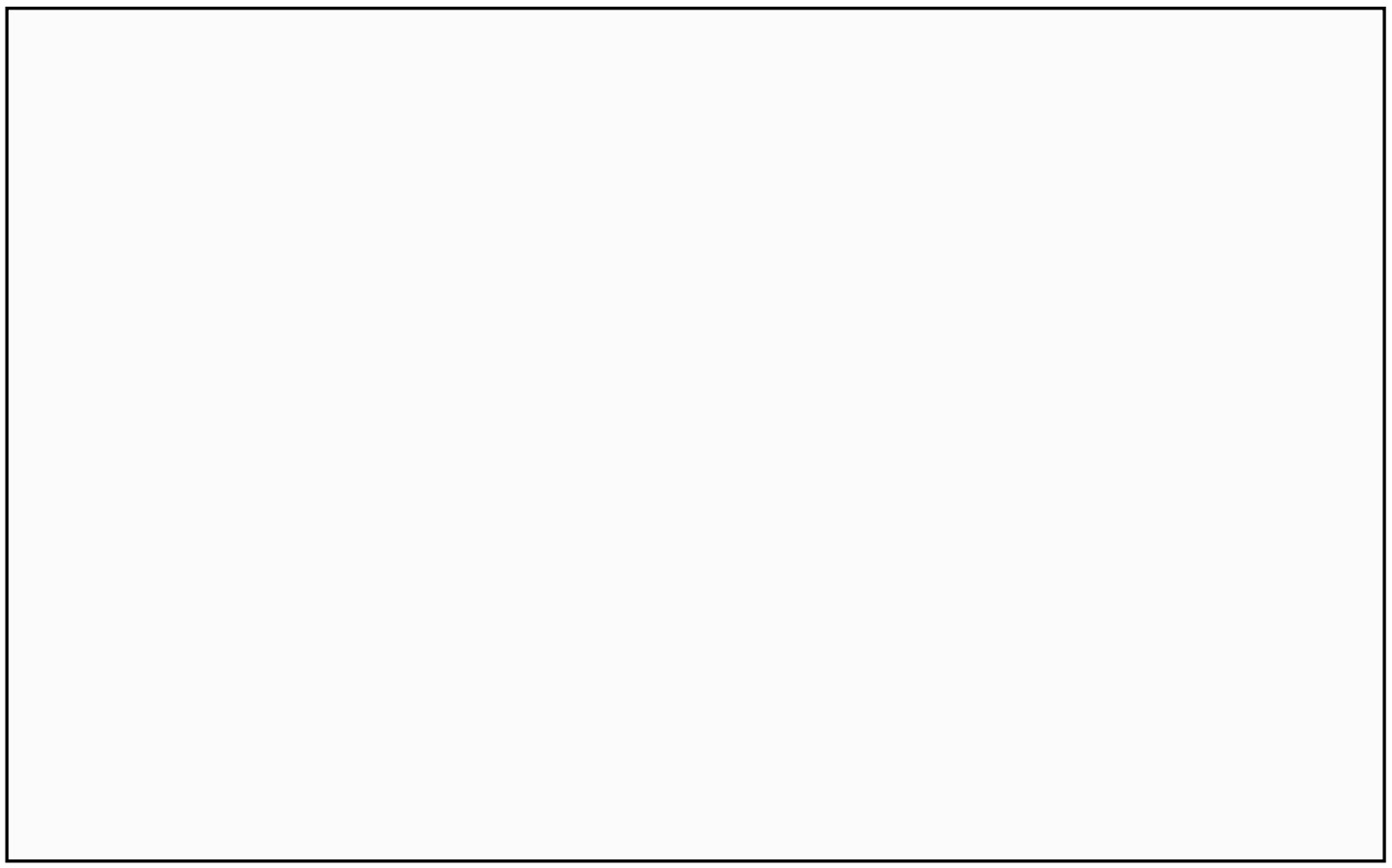

Table 1: A summary of the explicit scaling relations used to calculate the experimental parameters from those of the HIBALL-II design. The dependencies of the scaling on the energy and the dimensions are shown separately.

1 "HIBALL-II: An Improved Conceptual Heavy Ion Beam Driven Fusion Reactor Study", KfK 3840, Kernforschungszentrum Karlsruhe (July 1985), pp.57-71.

${ }^{2}$ MacLaren, S. A., et al. "A High Brightness Ion Source for Heavy Ion Fusion" , Proceedings of the 1999 Particle Accelerator Conference, New York, 1999 2855-58.

${ }^{3}$ De Hoon, M. J. L. and E. P. Lee, "Simulation of the LBNL Scaled Final Focus Experiment", these proceedings. 